\title{
Los sentidos de la democracia en Bolivia, Ecuador y Venezuela en el siglo XXI
}

\author{
Sense of Democracy in Bolivia, Ecuador and Venezuela in the 21st \\ Century
}

\section{Juan Bautista Lucca Cintia Pinillos}

\section{Resumen}

El artículo focaliza en las novedades semánticas en torno la noción de democracia que aparecen en los procesos de reforma constitucional en la primera década del siglo XXI en Venezuela, Bolivia y Ecuador. En primer lugar, analiza cómo la incorporación de instrumentos de democracia directa se plantean como una instancia donde desarrollar nuevas prácticas democráticas diferentes (en su diseño o mecánica) a las de su versión liberal clásica. En segundo lugar, analiza en qué medida este anhelo de nuevos sentidos democráticos pone en tensión a los actores principales de la versión representativa clásica (los partidos políticos), abriendo espacio para nuevos sujetos o modalidades de representación. Finalmente, se observa, cómo en las grietas de la tradición liberal representativa de la democracia, estos casos proponen nuevos "sentidos" de la democracia que se condensan en el momento en el que el poder constituyente de las sociedades diseña una nueva carta magna o constitución.

\section{Palabras clave}

Ecuador; Venezuela; Bolivia; Democracia; Reforma Constitucional.

\begin{abstract}
This work analyzes the semantic developments around the notion of democracy that appear in the process of constitutional reform in the first decade of the century in Venezuela, Bolivia and Ecuador. First, it will analyze how the incorporation of direct democracy are presented as an instance where different developing new democratic practices (in design or mechanical) to its classic liberal version. Secondly, we will analyze to what extent this longing for new democratic ways tenses the main actors of classical representative version, what are the political parties, and to what extent this opens space for new subjects or modes of representation. Thus it will be seen how, in the cracks of representative liberal tradition of democracy, these cases seem to find new "ways" of democracy that clearly are condensed in the moment in which the constituent power wants to mold their policy planning and designing a new political constitution.
\end{abstract}

\section{Keywords}

Ecuador; Venezuela; Bolivia; Democracy; Constitutional Reform. 


\section{Presentación}

A partir de la década de 1980, en América Latina, la democracia, entendida en los estrechos márgenes del concepto de régimen político, era una causa que parecía ordenar el sentido de las acciones de los diversos actores durante los procesos en curso. Inclusive, para los intelectuales que trataban de entender y explicar los fenómenos de cambio político, la democracia política era una fórmula para salir del autoritarismo y arribar a umbrales de consolidación y niveles de calidad democrática en la región.

Sin embargo, la desilusión sobre los resultados de la vida política en democracia fue apareciendo tras los primeros años de la instauración de los nuevos regímenes en los distintos países. A pesar de que las elecciones se convirtieron en el camino exclusivo de acceso al gobierno, y aunque la mayor parte de los actores principales del juego político aceptaron las reglas de juego, las deudas de la transición mostraban los límites de la democracia política para pensar la profundización y calidad de los procesos de democratización (MORLINO, 2007).

Más allá de episodios aislados, los militares se mantuvieron excluidos de acciones directas en contra del régimen democrático. Sin embargo, en algunos países, la subordinación de los militares al poder civil continuó siendo un elemento difícil de alcanzar para los gobiernos electos, a la par que se vio postergado - o en algunos casos, clausurado - el juzgamiento por los crímenes del terrorismo de Estado o la democratización del diseño y estructuras administrativas del Estado (LUCCA y HERNÁNDEZ CRUZ, 2013).

La perdurabilidad de los enclaves autoritarios luego de las elecciones fundacionales en Chile, el maridaje entre políticos y militares que parecía estar disolviéndose en Paraguay en los últimos años, o la tardía llegada de la paz en algunos países centroamericanos en los años 90, entre otros, son algunos ejemplos de la fortaleza del pasado reciente en la nueva democracia en la región.

Esto convive con un sucesión de crisis de inestabilidad presidencial y movilización social que, aunque pusieron en entredicho la representatividad democrática, no horadaron la continuidad de la democracia, como pudo verse en las salidas anticipadas de Ricardo Alfonsín (1989) y Fernando De la Rúa (2001) en Argentina, Fernando Collor de Mello (1992) en Brasil, Alberto Fujimori (1992) en Perú, Carlos Andrés Pérez (1993) en Venezuela, Ernesto Samper (1996) en Colombia, Abdalá Bucaram (1997) en Ecuador, o Raúl Cubas Grau (1999) en Paraguay, entre otros (PÉREZ LIÑÁN, 2009). 
Una evaluación de los procesos de democratización en la región, realizada desde los primeros años del nuevo milenio, muestra un panorama de claros y oscuros, que incluyen: una creciente movilización social por un lado, pero serias deficiencias en el ejercicio efectivo de la ciudadanía y la inclusión política por el otro (ANSALDI, 2007); un cambio en el sentido de la política pública en mucho de los países que revisaron su herencia neoliberal por un lado, y un déficit en la eficacia gubernamental para conseguir nuevos objetivos de solidaridad social, administrando recursos escasos por el otro (MONEDERO, 2012, p. 76); la (re)incorporación de nuevos sujetos sociales al mundo del trabajo y de la inclusión social por un lado, acompañados de retrocesos en materia de democratización de derechos sociales y la distribución de la riqueza, especialmente en países de mayores ingresos per cápita, por el otro (LECHNER, 1988, p. 32; HOLSTON, 2007). En este panorama, la democracia muestra también su ambivalencia, al haber logrado posicionarse como idea ordenadora del horizonte social y político por un lado, pero presentar una configuración acotada, especialmente a su versión liberal representativa, que no termina de seducir plenamente a los latinoamericanos ni permite comprender la complejidad de los procesos de construcción de la democracia en perspectiva comparada en la región (HAGOPIAN, 2000).

Este complejo escenario de la democracia latinoamericana en los inicios del nuevo siglo, devela una problemática de raíces profundas: la necesidad de repensar el alcance explicativo de dicho concepto, de revisar los atributos definitorios del mismo para poder viajar por el territorio, y en cierta medida apelar a diversas estrategias para dar cuenta de los sentidos que adjetivan el sustantivo democracia en la realidad latinoamericana (COLLIER y LEVITZKY, 1998).

Ahora bien, si durante los ochenta y noventa del siglo XX, al concepto liberal representativo de democracia se le anexaron o quitaron atributos y adjetivos en vistas a explicar mejor la diversidad empírica de este régimen político en la región, los primeros años del siglo XXI reabren el debate en torno a los sentidos del sustantivo democracia, especialmente en procesos constitucionales como los de Venezuela (1999), Bolivia (2009) y Ecuador (2008). Estas experiencias, complejas y diversas entre sí, tienen en común presentar una fisura en el sentido de la democracia en su versión liberal representativa, que imperó durante gran parte del siglo XX. Por ende, en este ensayo, se llevará a cabo un análisis histórico comparativo de carácter cualitativo de las cartas constitucionales de los tres países, focalizando en dos aspectos donde se evidencia una primacía de un nuevo sentido democrático: 
a) Cómo la incorporación de instrumentos de democracia directa se plantean como una instancia donde desarrollar nuevas prácticas democráticas diferentes (en su diseño o mecánica) a las de su versión liberal clásica;

b) En qué medida este anhelo de nuevos sentidos democráticos pone en tensión los actores principales de la versión representativa clásica, cuales son los partidos políticos, y en qué medida esto abre el espacio para nuevos sujetos o modalidades de representación.

La hipótesis en la que se sustenta esta reflexión parte de aseverar que junto a la recuperación de la democracia en la región como horizonte ordenador del sentido de lo público, especialmente hacia la década de 1980, se produce la imposición de una versión radical del sentido liberal de la política (comúnmente denominada la neoliberal), que ponía el acento en el carácter individualista de la praxis política, que horadó los actores clásicos de la democracia representativa como son los partidos políticos, pero también toda otra construcción de sujetos colectivos tradicionales, como por ejemplo los sindicatos. Sin embargo, en esta "desertificación neoliberal" surgieron espacios de fuga, donde el sentido de la democracia fue puesto en debate, tensión y reconfiguración, especialmente a la luz de las experiencias de movilización de nuevos sectores sociales que, anteriormente, no participaban de la palestra pública o inclusive la incorporación de mecanismos de participación ciudadana de nuevo cuño. Es a partir de esta reacción a la democracia (neo)liberal que se configuran nuevos entramados de poder popular constituyente, que buscó la innovación en torno a la democracia y lo democrático.

\section{Democracia directa}

A partir de los procesos de transición desde regímenes autoritarios en la década del '80 del siglo pasado, las reformas constitucionales han constituido un evento frecuente en los países de América Latina. Como apunta Gabriel Negretto (2009, p. 38), todos los países de la región han reformado o reemplazado sus Constituciones, así como también leyes secundarias que regulan aspectos claves tanto del régimen político o del sistema electoral. Una nota generalizada de estos procesos es la introducción de mecanismos de democracia directa: plebiscitos, iniciativas populares, referéndums, referéndums revocatorios, iniciativas consultivas, entre otras (ZOVATTO, 2007, p. 39).

Un estudio comparativo de las consecuencias políticas de la incorporación constitucional de mecanismos de democracia directa resultaría vacuo al considerar solamente la letra constitucional o el carácter simbólico de dicha innovación, ya que, en general estas innovaciones obedecen tanto a un contexto socio-político de 
ampliación de derechos y de participación, como también por voluntad pública de ciertos actores sociales y políticos que pujan por la inclusión de estos instrumentos para una nueva praxis política (DE SOUSA SANTOS y AVRITZER, 2004).

Las tres reformas constitucionales estudiadas fueron el resultado de referendos nacionales, ya que las Constituciones anteriores y sus correspondientes modificaciones de la década del '90 (Venezuela 1961, Ecuador 1998 y Bolivia 1967) preveían este mecanismo para la modificación de la Carta Magna.

Para abordar en detenimiento el caso de Bolivia, resulta imprescindible revisar el proceso de reforma que se inicia a mediados de la década de 1980. Fernando Mayorga (2009) propone una periodización interesante, que divide este período de más de dos décadas en tres momentos de ampliación democrática:

1. Entre 1985 y 2003, se da una situación de "democracia pactada" y reforma "desde arriba", habida cuenta de la sucesión de cinco gobiernos de coalición, ya que los principales partidos políticos del período, MNR, MIR y ADN, no solo concentraron dos tercios del apoyo electoral, sino también aplicaron una lógica consociativa. En este período, se sucedieron la reforma constitucional parcial de 1994, el rediseńo de la ley de partidos políticos y de participación popular. Esta última ley otorgaba poder a las instancias locales, elemento que, junto con la introducción de las circunscripciones uninominales, favorecieron la aparición de una nueva arena para la praxis política, en la que pudo plasmarse una subcultura política sin representación hasta entonces, como fue, por ejemplo, la génesis del movimiento cocalero, indigenista y por ende del MAS.

2. La segunda ampliación es definida por Mayorga como crisis política y presión "desde abajo". La misma tiene sus antecedentes en las protestas sociales del año 2000, que precedieron una disminución electoral de los partidos políticos tradicionales y el crecimiento de las nuevas fuerzas políticas. El fin de la "democracia pactada" puede ubicarse en 2003, con la renuncia del presidente Sánchez de Lozada, en un marco de polarización y crisis política. Lo que define este proceso político es un doble juego de impugnaciones, por un lado con las demandas del movimiento campesino e indigenista que promovían la asamblea constituyente y la nacionalización de los hidrocarburos, y por otro con los movimientos cívicos regionales, demandando una mayor autonomía departamental. Ambas presiones "desde abajo" configuran el telón de fondo de la segunda reforma constitucional parcial de 2004. En este contexto, dos innovaciones importantes son el fin del monopolio de la representación para 
los partidos políticos - elemento que se analizará más adelante - y la elección directa de los prefectos como máximas autoridades del nivel departamental. Además, se incorporan el referéndum, la iniciativa legislativa ciudadana y la asamblea constituyente, transformando las pautas del proceso de toma de decisiones y de reforma constitucional (ROMERO BALLIVIÁN, 2008, p. 159).

3. La tercera ampliación se abre cuando el MAS triunfa en las elecciones de finales de 2005. Este momento es definido por Mayorga como de polarización política y proceso constituyente, y culmina en la aprobación de la Constitución de 2009.

En el marco de este desarrollo histórico del proceso constituyente del poder en Bolivia, es importante observar cómo la Constitución de 2009 introduce una torsión en los sentidos de la democracia. Cabe tener en cuenta que hasta las reformas precedentes (2002 y 2004) - especialmente en el artículo 1, denominado "Clase de Estado y Forma de Gobierno" - la democracia estaba pensada tanto como una forma de gobierno (representativa y participativa), como también un adjetivo central del formato del Estado boliviano. Sin embargo, tras la reforma, la noción de democracia se expande y, a pesar de preservar su componente liberal representativo y reforzar su componente participativo y directo, incorpora una nueva forma de manifestación: "democracia comunitaria".

Art. 11: La democracia se ejerce de las siguientes formas, que serán desarrolladas por la ley:

1. Directa y participativa, por medio del referendo, la iniciativa legislativa ciudadana, la revocatoria de mandato, la asamblea, el cabildo y la consulta previa. Las asambleas y cabildos tendrán carácter deliberativo conforme

a

Ley.

2. Representativa, por medio de la elección de representantes por voto universal, directo y secreto, conforme a Ley. 3. Comunitaria, por medio de la elección, designación o nominación de autoridades y representantes por normas y procedimientos propios de las naciones y pueblos indígena originario campesinos, entre otros, conforme a Ley (BOLIVIA, 2009).

Si se analiza el caso de Ecuador, resulta conveniente poner en contexto a la actual Constitución. Entre 1830 y 2005, estuvieron vigentes 18 constituciones, mientras que, desde la apertura del último período democrático, fueron solo dos las Cartas Magnas vigentes: la de 1979 y la de 1998. 
Así es posible afirmar que Ecuador ha disfrutado de un considerable número de constituciones, que desde el inicio han abrazado el ideal republicano y democrático, aunque con sustanciales restricciones hasta la Constitución de 1979 (hasta 1852 eran ciudadanos sólo los propietarios y profesionales de alto nivel, y hasta la Constitución de 1979, los analfabetos estaban excluidos del derecho al voto). Las profundas reformas introducidas en 1998 consagraron derechos de las minorías sociales, de las poblaciones indígenas y afro ecuatorianas, para el medio ambiente y los recursos nacionales. Es a partir de esta constitución, muy cuestionada por los partidos de derecha, y que casi implicó por su alcance una nueva constitución - que, por primera vez, el Ecuador se reconoce como país multiétnico y pluricultural (PAZ y CEPEDA, 2007, p. 296).

Además, el ordenamiento constitucional de 1998 contemplaba las tres formas básicas de democracia directa: el referendo, la iniciativa legislativa y la revocatoria de mandato. El referéndum y la iniciativa legislativa ya estaban presentes en la Constitución de 1978, y la revocatoria se incorporó en la Constitución de 1998 (PACHANO, 2008, p. 145). Si bien el referéndum ha sido aplicado por diversos gobiernos, la revocatoria de mandato no ha sido utilizada, y la agitada vida política ecuatoriana de los últimos años ha mostrado la recurrencia a la resolución de los conflictos por la acción directa.

En la Constitución vigente de Ecuador, se dedica la Sección IV a la democracia directa; en la misma, se establecen los mecanismos de iniciativa popular (Art. 103), la consulta popular (Art. 104) y la revocatoria de mandato para todas las autoridades elegidas popularmente (Art. 105). Es interesante notar la evolución que se le da al estatuto de la participación dentro de la constitución, ya que si en la de 1998 estaba incluida en el Título IV - De La Participación Democrática - dentro del apartado "De otras formas de participación democrática", como anexo a la participación electoral; en la constitución de 2008 la noción de participación en democracia se amplía, se profundiza y se caracteriza, colocando a los mecanismos de democracia directa en un estatuto similar al de las demás formas de participación, incluida la electoral.

Si se toma en cuenta el caso de Venezuela, la Constitución de 1961 puede definirse como un ejemplo clásico de Constitución representativa, ya que sólo contemplaba las figuras del referéndum consultivo nacional para aprobar reformas a la Constitución y la iniciativa legislativa, dos herramientas relativamente comunes en las Constituciones latinoamericanas del siglo XX. En las modificaciones que se dieron 
en la década del '80, se incluyeron los referendos sobre las gestiones de alcaldes y gobernadores, así como otro tipo de consultas menores.

En un contexto histórico previo de desestructuración del sistema político articulado por el Pacto de Punto Fijo, a mediados del siglo XX, donde los partidos tradicionales fueron desplazados del centro de la escena política - y donde el protagonismo ciudadano ganó las calles luego de décadas de una "excepcional estabilidad política" que contrastaba con la mayor parte de los procesos nacionales del resto de los países de la región, la profundización de instrumentos de democracia directa, introducidos a partir de la Constitución propiciada por el gobierno de Hugo Chávez, parecía venir a resolver una deuda pendiente de la democracia liberal y representativa.

En este sentido, la Constitución venezolana de 1999 contempla el instrumento de democracia directa menos difundido que es la revocatoria de mandato establecida para todos los cargos y magistraturas de elección popular (Art. 72), incluyendo el cargo de presidente de la nación, que pudo verse en práctica en el 2004 (KORNBLITH, 2008).

Ahora bien, es posible superponer otra mirada a aquella que señala las ventajas de la Constitución de 1999 y el proceso político reciente como instancia superadora de la desarticulación del sistema político del puntofijismo. Miriam Kornblith (2008) argumenta que más allá de la valoración positiva que ha implicado el reconocimiento de estas instancias participativas como derechos ciudadanos, no quita que ellas han fortalecido la articulación entre el líder y el pueblo en desmedro de otros actores centrales de la vida política, apreciándose un proceso de subordinación y degradación de instrumentos convencionales de la democracia (p. 128).

En este sentido, Alicia Lissidini (2008, p. 13) señala que los mecanismos incorporados en las últimas décadas en las Constituciones latinoamericanas pueden ser analizados como herramientas políticas con capacidad de promover tanto la participación y el involucramiento de los ciudadanos en los asuntos públicos, como la expansión de la influencia de las prerrogativas de los ejecutivos, en detrimento de los instrumentos propios de la democracia representativa - sobre todo los partidos políticos -, favoreciendo así procesos delegativos. Por ende, no solo debe realizarse la distinción entre aquellos instrumentos que tienen vocación proactiva - como la iniciativa legislativa - de aquellos otros - como el veto o la revocatoria de mandatos que tienen efectos reactivos, sino también observar con claridad, como destaca David Altman (2005, p. 225), el origen (Poder Político o Sociedad Civil) y el uso político 
(auto-legitimación del poder político o control-censura del mismo) de estos mecanismos.

En resumidas cuentas, cabe señalar que las herramientas constitucionales van a tener diverso alcance a través de los procesos políticos en los que se estudie su aplicación. Si se observan otros contextos históricos de la historia latinoamericana reciente, los plebiscitos han servido para impedir la continuidad de regímenes autoritarios (como el caso de Uruguay o Chile), atentando contra los propios objetivos de aquellos que convocaban la consulta popular.

Así, los instrumentos de democracia directa pueden contribuir a cristalizar situaciones políticas preexistentes de deslegitimación de la democracia representativa, pero pueden también contribuir a delinear diversas modalidades de ejercicio del poder en el futuro, impactando de manera diferenciada sobre los mismos actores que promovieron su implementación.

\section{Transformación de la representación politico partidaria}

En todas las constituciones de tipo liberal representativo, existe un actor preponderante en la vinculación entre sociedad y estado: los partidos políticos. Por ende, no es un detalle menor, que en las diversas reformas constitucionales analizadas, el rol de los mismos sea puesto en entredicho, dando lugar a experiencias de innovación. Mientras que la Constitución Argentina de 1994 establece que los partidos políticos son instituciones fundamentales del sistema democrático (Art. 38), promoviendo el monopolio de los mismos en la presentación de candidatos en las elecciones; las tres Constituciones en estudio de Bolivia, Ecuador y Venezuela les reservan un lugar de menor centralidad en el sistema político.

En general, estas modificaciones vienen de la mano de virajes que se explican tanto por el contexto de crisis de los partidos y sistemas de partidos en cada uno de los países, como también por el signo de la política de corte neoliberal. En este sentido, el fin de siglo resultó, para la región, un momento de gran cambio y reconversión partidaria, puesto que se produjo el viraje desde un modelo social de acumulación focalizado en la industrialización por sustitución de importaciones, en la que el Estado oficiaba de articulador o centro de la economía y la política, hacia un esquema neoliberal en que el Mercado se erigía como amo y señor en el dictado de los valores imperantes en las esferas de la política (imprimiendo la apoliticidad y aversión al conflicto), en la economía (conduciendo a la apertura indiscriminada y la desestructuración de las economías nacionales) y la sociedad (produciendo el desmembramiento del tejido social) (ROBERTS, 2002). 
En este sentido, el neoliberalismo en América Latina tuvo un enorme impacto en "achicar" al Estado y correrlo del centro de la escena como productor de sociabilidad. Paradójicamente, esta simplificación del Estado se daba justamente en el instante en que desde la sociedad se plantaban una creciente multiplicación de las demandas así como también una diversificación y fragmentación de los grupos sociales - incluso a nivel territorial - que, como señala Norbert Lechner (1992), hacía necesario generar el sentido del orden que supone el juego político y no la preponderancia de la lógica de oportunidades que propone el Mercado.

En este panorama, se produce dos fenómenos encontrados, que son de enorme relevancia para comprender la nueva topografía de la representación política en la región: un fuerte desapego al corporativismo y la representación partidaria en la gran mayoría de los casos (con contadas excepciones como en Chile o Uruguay), y en contrapartida un fuerte descontento social y el crecimiento de nuevas demandas ciudadanas y una fuerte activación o movilización social.

Tal y como lo planteare Frances Hagopian (2000), los albores del siglo XX, en América Latina, dan cuenta de una segunda "resurrección de la sociedad civil", lo cual tornó a la democracia en un entramado más incluyente, pero no necesariamente más representativa, dado que los nuevos movimientos sociales que surgieron pueden haber movilizado segmentos más amplios de la ciudadanía que los partidos en el pasado, pueden haber expresado mejor aquellos déficits políticos estructurales que nuevas fuerzas partidarias, o inclusive erigirse en un espacio de prueba y error para el juego democrático, pero necesariamente chocaban contra los límites del acceso a la representación política, convirtiéndose por ende en algún punto en estructuras innovadoras, pero poco eficaces. Por ello, en algún punto, esta pujanza e innovación de los nuevos movimientos sociales de finales del siglo XX latinoamericano fue un enorme acicate, no solo para pensar los canales de participación, como se vio previamente, sino también para repensar - inclusive en el plano constitucional - a la representación política (MONEDERO, 2011, p. 176 y 177).

Si se pone el acento en la comprensión del caso de Bolivia en este punto, es necesario reconocer el derrotero de la transformación de la representación del sistema de partidos, del ordenamiento institucional, así como también de sus iniciativas innovadoras, como la incorporación de las Agrupaciones Ciudadanas (AC) y los Pueblos indígenas (PI) como nuevos actores de la representación política.

Desde 1985, en Bolivia, nos encontramos con una sucesión de "pactos de gobernabilidad" entre los principales partidos, configurando lo que, para Jorge Lazarte (2006, p. 102), puede denominarse como un "sistema de tres más cuatro"; es 
decir, tres partidos principales que articulaban la disputa (Movimiento Nacional Revolucionario - MNR -, Acción Democrática Nacionalista - ADN - y el Movimiento de Izquierda Revolucionario - MIR) y cuatro organizaciones partidarias que oficiaban de socios de contingencia para la formación de mayorías para elegir al presidente de turno.

Sin embargo, esta dinámica de colusión mostraba, en paralelo, un desgaste de las organizaciones partidarias en su enraizamiento social, especialmente desanclándolo de la marginada - pero mayoritaria - población indígena. Para tratar de revertir este desencuentro entre la ciudadanía social y los partidos políticos, se llevaron adelante innumerables reformas, como por ejemplo: en 1995, se incluyó la elección directa de la mitad de los diputados en circunscripciones uninominales; en 1999, se incorpora a la ley de partidos la obligatoriedad de utilizar mecanismos electivos para la selección de candidatos; en el año 2002 - en un marco de fuerte descontento y presión social -, se suma la figura de las AC como nuevo actor capaz de postular candidatos a los cargos electivos; y en febrero de 2004 - cuando la inestabilidad social y política era un hecho dado -, se pone en un plano de paridad en términos de la representación política a los partidos, las AC y los PI.

Este derrotero de cambios en la forma de la representación política no puede entenderse sin tomar en cuenta que lo que originalmente era malestar ciudadano acumulado frente a la elite político partidaria tradicional y sus formas de la política representativa se convirtió en efervescencia y movilización social entre el 2002 y 2005, que obstaculizó todo intento por mantener en funcionamiento la política de pactos (evidentes las renuncias de Sánchez de Losada en 2003 y Mesa en 2005) y que dio paso a un nuevo sentido de la política anclado en el conflicto (manifiesto en la Guerra del Agua, del Gas y la Coca), donde primaron nuevos actores partidarios que desafiaban el status quo e incorporaban nuevas elites y ciudadanía (como puede verse claramente en el crecimiento electoral del Movimiento al Socialismo - MAS).

En este sentido, la década de 2000, en Bolivia, es una coyuntura de replanteo de la representación partidaria y de innovación política, que puede verse evidente, el primer punto, en los datos que ofrece Latinobarómetro sobre la importancia de los partidos para la política, y el segundo aspecto, en la morfología que adquiere la nueva constitución de 2009 en cuanto a los actores representativos. Respecto al primer aspecto, como puede advertirse en el cuadro a continuación, entre el ańo 2001 y 2008 más de la mitad de los bolivianos encuestados consideran que la democracia puede funcionar sin partidos, lo cual da cuenta de la crisis de los partidos tradicionales, pero también, en un primer momento, del desapego a la figura de los 
partidos in totto evidente en la incorporación de nuevas figuras como la AC y PI, aspectos que - junto a la emergencia del MAS y Evo Morales - configuran una torsión en la representación de la ciudadanía.

Tabla 1 - Bolívia

\begin{tabular}{l|l|l|l|l|l|l|l|l|l|l|l}
\hline & 1997 & 2000 & 2001 & 2002 & 2005 & 2006 & 2008 & 2009 & 2010 & 2011 & 2013 \\
\hline $\begin{array}{l}\text { Sin } \\
\text { partidos } \\
\text { políticos no } \\
\text { puede } \\
\text { haber } \\
\text { democracia }\end{array}$ & $0 \%$ & $7 \%$ & $2 \%$ & $2 \%$ & $1 \%$ & $8 \%$ & $5 \%$ & $1 \%$ & $1 \%$ & $7 \%$ & $4 \%$ \\
\hline $\begin{array}{l}\text { La } \\
\text { democracia } \\
\text { puede } \\
\text { funcionar } \\
\text { sin partidos }\end{array}$ & $1 \%$ & $0 \%$ & $4 \%$ & $6 \%$ & $6 \%$ & $2 \%$ & $1 \%$ & $8 \%$ & $6 \%$ & $8 \%$ & $4 \%$ \\
\hline $\begin{array}{l}\text { No } \\
\text { contesta }\end{array}$ & $\%$ & $2 \%$ & $\%$ & $\%$ & $\%$ & $\%$ & $\%$ & $\%$ & $\%$ & $\%$ & $\%$ \\
\hline No sabe & $\%$ & $\%$ & $1 \%$ & $\%$ & $1 \%$ & $\%$ & $1 \%$ & $\%$ & $2 \%$ & $3 \%$ & $1 \%$ \\
\hline \begin{tabular}{l} 
(N) \\
\hline
\end{tabular} & 96 & .080 & .075 & .242 & .200 & .200 & .200 & .200 & .200 & .200 & .400 \\
\hline
\end{tabular}

Fonte: Elaboración propia con base en el análisis online disponible en Latinobarómetro (2015).

Este cambio del sentido de la representación política boliviana no solo se manifiesta por el cambio en las siglas partidarias, sino más bien por el intento de repensar el diseño y la dinámica electoral, al punto tal que, en la Constitución Boliviana de 2009, en su artículo 210, establece la igualdad ante la ley de diversas organizaciones democráticas para canalizar la presentación de candidatos en las elecciones:

Art. 210 Las candidatas y candidatos a los cargos públicos electos, con excepción de los cargos elegibles del Órgano Judicial y del Tribunal Constitucional Plurinacional serán postulados y postuladas a través de las organizaciones de las naciones y pueblos indígena originario campesinos, las agrupaciones ciudadanas y los partidos políticos (BOLIVIA, 2009).

Si tomamos en cuenta el caso de Ecuador, cabe señalar que, desde la recuperación democrática, este país ha vivido envuelto en la marańa de la discontinuidad política y fragilidad partidaria evidente en la habitual salida anticipada de sus presidentes. En este sentido, Simón Pachano apunta que, desde 1979 hasta la 
llegada de Rafael Correa, los partidos y sistema de partidos ecuatorianos adolecieron de: la fragmentación, evidente en el alto número de partidos que participan en el Congreso y la magnitud variable de los bloques que allí se conformaron; la volatilidad, que se manifiesta en que los partidos no lograron repetirse más de una vez en la presidencia; la polarización, con el agravante de que los extremos ideológicos son ocupados por partidos de relevancia; la dispersión territorial de los partidos en la Costa, o la Sierra y, en menor medida, en la región de la Amazonia (PACHANO, 2004, p. 71).

Ahora bien, esta discontinuidad política, a pesar de la persistencia y relevancia en el tiempo de algunos partidos, como los Social Cristianos, la Izquierda Democrática, el Roldoista Ecuatoriano y la Democracia Popular, adquirió características diversas si se desagrega este período 1979-2008, tomando en cuenta el tipo de juego político imperante, las reglas de juego constitucionales que lo determinan y las particularidades de los actores políticos y partidarios intervinientes. En este sentido, Santiago Basabe-Serrano (2009, p. 402-404) propone subdividir el período en tres momentos: a) 1979-1997; b) 1997-2006; y c) 2006 en adelante. El primero de estos lapsos temporales esta signado por el equilibrio inestable que se produce en la pugna entre los actores legitimados electoralmente y otros actores con capacidad de veto (como los militares o las elites económicas), entre las elites regionales de la Costa y la Sierra, y entre los actores políticos tradicionales y aquellos que representan a nuevas minorías políticamente significativas, como por ejemplo los indígenas.

Tras esta situación de empate, según el autor, se abre un segundo momento político, con la Constitución de 1998, donde la debilidad institucional y fragmentación política busca ser subsanada con estrategias institucionales que refuercen la figura y prerrogativas del presidente, por sobre otras instancias y poderes del Estado; sin embargo, estos resortes institucionales no son suficientes como para detener el espiral destituyente que llevó a la caída de los presidentes Jamil Mahuad en el año 2000, o Lucio Gutiérrez, en el 2005, ni para poner freno a un fuerte desprestigio de los partidos políticos - como puede verse en el siguiente cuadro con base en los datos de Latinobarómetro - o la radicalización de un discurso "antipartidario" y "anti-política" con enorme sustrato histórico en Ecuador (BASABESERRANO, PACHANO y MEJÍA ACOSTA, 2010, p. 79). 
Tabla 2 - Ecuador

\begin{tabular}{l|l|l|l|l|l|l|l|l|l|l|l}
\hline & 1997 & 2000 & 2001 & 2002 & 2005 & 2006 & 2008 & 2009 & 2010 & 2011 & 2013 \\
\hline $\begin{array}{l}\text { Sin } \\
\text { partidos } \\
\text { políticos no } \\
\text { puede } \\
\text { haber } \\
\text { democracia }\end{array}$ & $4 \%$ & $9 \%$ & $1 \%$ & $9 \%$ & $2 \%$ & $5 \%$ & $9 \%$ & $4 \%$ & $2 \%$ & $0 \%$ & $4 \%$ \\
\hline $\begin{array}{l}\text { La } \\
\text { democracia } \\
\text { puede } \\
\text { funcionar } \\
\text { sin partidos }\end{array}$ & $8 \%$ & $9 \%$ & $1 \%$ & $9 \%$ & $2 \%$ & $5 \%$ & $2 \%$ & $4 \%$ & $8 \%$ & $1 \%$ & $5 \%$ \\
\hline $\begin{array}{l}\text { No } \\
\text { contesta }\end{array}$ & $\%$ & $\%$ & $\%$ & $\%$ & $\%$ & $\%$ & $\%$ & $\%$ & $\%$ & $\%$ & $\%$ \\
\hline No sabe & $\%$ & $\%$ & $\%$ & $\%$ & $4 \%$ & $\%$ & $8 \%$ & $1 \%$ & $\%$ & $\%$ & $0 \%$ \\
\hline \begin{tabular}{l} 
(N) \\
\hline
\end{tabular} & .200 & .200 & .200 & .200 & .200 & .200 & .200 & .200 & .200 & .200 & .400 \\
\hline
\end{tabular}

Fonte: Elaboración propia con base en el análisis online disponible en Latinobarómetro (2015).

El tercer período se inicia el 26 de noviembre de 2006 con el triunfo de Rafael Correa, y se corporiza en la Constitución de 2008 y en los ribetes que adquiere la "Revolución Ciudadana" en Ecuador. Particularmente el proceso constitucional que se abre en el 2008 no es la expresión única de Correa por renovar y reformar el sistema político, sino más bien la culminación de una gran cantidad de intentos infructuosos previos por modificarla; sin embargo, la forma y dinámica que adquiere este proceso (especialmente con las irregularidades conocidas como la génesis de los diputados de los manteles) y gran parte de la orientación de la misma sí es atribuible a Correa y sus aliados coyunturales.

En lo que atañe a los partidos políticos y la representación política, es interesante señalar, cómo en la Constitución de Montecristi de 2008, especialmente en el artículo 108, se habla de organizaciones políticas reconociendo tanto a partidos políticos como a movimientos políticos en tanto organizaciones públicas no estatales que encarnan la pluralidad política y que pueden canalizar las distintas opciones en la contienda electoral. Ahora bien, esta pluralidad que manifiesta la constitución entre partidos que deben necesariamente propender a ser una expresión nacional y los movimientos políticos que deben tener al menos $1,5 \%$ del padrón, si bien puede llevar a pensar a estos últimos como la vía inicial de fuga al clima de desprestigio de los partidos que precedió a la llegada de Correa, en realidad es el resabio de la incorporación a la disputa electoral de la figura del "independiente" en la 
Constitución de 1998. En todo caso, lo que la Constitución de 2008 viene a proponer (e incluso adquiere mayor evidencia en la Ley Orgánica Electoral de 2009) es un movimiento inverso, de acotar el ingreso desmedido de los outsiders o, en todo caso, compelerlos a que una vez que irrumpen en el sistema y persisten exitosamente (más del 5\% de los votos en elecciones pluripersonales según el artículo 110), necesariamente adquieran la institucionalidad de un partido político. En este sentido, la constitución ecuatoriana, si bien pone en entredicho la representación política partidaria al igual que los otros dos casos estudiados, no escapa al intento de remendar institucionalmente la fragilidad partidaria y la volatilidad de los ciudadanos vivida por décadas.

En el caso de Venezuela, la mixtura de un contexto político partidario en franco cambio, la irrupción de una nueva forma de la política, y el declive de los partidos históricos, fueron los elementos para comprender cómo la representación política partidaria no solo se transforma, sino que adquiere también una morfología diferente para la tradición histórica de ese país.

Entre 1958 y 1973, la estabilidad política y la paz social eran el producto del pacto de Punto Fijo (1958) entre Acción Democrática (AD), la Unión Republicana Democrática (URD) y el Comité de Organización Política Electoral Independiente (COPEI), que se plasmó en la Constitución de 1961 como epicentro de la legitimación de la resolución del conflicto político por la vía electoral, y no por la apelación al uso de la fuerza o de los militares como garantes; lo cual puede verse claramente en el Artículo 3 y 4 de la Constitución de 1961:

Art. 3. El gobierno de la República de Venezuela es y será siempre democrático, representativo, responsable y alternativo.

Art. 4. La soberanía reside en el pueblo, quien la ejerce, mediante el sufragio, por los órganos del Poder Público (VENEZUELA, 1961).

La entronización de este sistema político fuertemente partidocrático tenía como finalidad neutralizar - tanto como fuera posible - cualquier esfuerzo desestabilizador, pero también de incorporación de terceras fuerzas políticas (como el Movimiento al Socialismo en los setentas). Según Ramos Jiménez (2002, p. 388), este esquema partidario llevó a $\mathrm{AD}$ y COPEI hacia una cartelización del Estado y la configuración de estructuras partidarias menos ideologizadas, con una estructura dirigencial muy cerrada, pero, en contrapartida, con un anclaje social cada vez más débil. El tiro de gracia a esta situación será la elección, por segunda vez, de Carlos 
Andrés Pérez, quien, a pesar de su gran apoyo electoral inicial, optará por desentenderse de su partido y optar por un entorno de gobierno de corte tecnocrático con una orientación de política pública de corte neoliberal (RAMOS JIMÉNEZ, 1999, p. 38).

Esto generó una reacción en cadena de características múltiples, ya que daba pie al crecimiento de un espíritu antipartidista (del cual Pérez era paradojalmente el primer exponente), pero también al auge de la movilización y protesta social en descontento con la clase política (el Caracazo en 1989 y dos irrupciones militares en 1992), y el surgimiento de nuevos partidos y liderazgos, como los de La Causa R o Rafael Caldera, que aprovecharon esta crisis de representación partidaria a nivel nacional y nuevos espacios de representación subnacional que la descentralización administrativa había incorporado en la enmienda constitucional de 1980.

Ahora bien, el gobierno de Caldera (1994-1999) no fue una transición entre el viejo esquema bipartidista y una nueva estructura de representación política partidaria, sino la continuidad de la fortaleza del bipartidismo especialmente en el plano legislativo; con lo cual, la elección de 1998 era una coyuntura donde pervivían muchos rasgos de viejas tradiciones representativas, un sentido de hartazgo generalizado, y pocas expresiones políticas que podían encarnar por sí solas un carácter refundacional de la representación.

Con excepción de aquellos ciudadanos que manifestaron su hartazgo a través de la abstención electoral (más de un tercio de los electores), o su preferencia por terceras fuerzas que, desde 1960, no lograron crecer dentro de la partidocracia venezolana, la otra salida posible al hastío de la clase política vinculada a $A D$ y COPEI era la del Movimiento V República encabezado por Hugo Chávez Frías.

Ahora bien, más allá del análisis posible sobre las causales del éxito electoral de Chávez, lo que queda a las claras si se toma en cuenta el derrotero histórico aquí planteado, pero también el creciente descontento hasta el año 2000 que los venezolanos manifiestan hacia los partidos políticos según Latinobarómetro a continuación, es el fracaso a finales de siglo del proyecto iniciado en 1958 y con esto la apertura de un nuevo interregno de reformulación institucional, de redefinición de los sentidos de la democracia, pero especialmente de reconfiguración de las formas de la representación política. 
Tabla 3 - Venezuela

\begin{tabular}{l|l|l|l|l|l|l|l|l|l|l|l|l}
\hline & $\mathbf{1 9 9 5}$ & $\mathbf{1 9 9 7}$ & $\mathbf{2 0 0 0}$ & $\mathbf{2 0 0 1}$ & $\mathbf{2 0 0 2}$ & $\mathbf{2 0 0 5}$ & $\mathbf{2 0 0 6}$ & $\mathbf{2 0 0 8}$ & $\mathbf{2 0 0 9}$ & $\mathbf{2 0 1 0}$ & $\mathbf{2 0 1 1}$ & $\mathbf{2 0 1 3}$ \\
\hline $\begin{array}{l}\text { Sin partidos } \\
\text { políticos no } \\
\text { puede haber } \\
\text { democracia }\end{array}$ & $5 \%$ & $0 \%$ & $8 \%$ & $4 \%$ & $5 \%$ & $6 \%$ & $9 \%$ & $6 \%$ & $0 \%$ & $0 \%$ & $1 \%$ & $8 \%$ \\
\hline $\begin{array}{l}\text { La democracia } \\
\text { puede } \\
\text { funcionar sin } \\
\text { partidos }\end{array}$ & $7 \%$ & $2 \%$ & $9 \%$ & $6 \%$ & $5 \%$ & $4 \%$ & $3 \%$ & $0 \%$ & $3 \%$ & $4 \%$ & $2 \%$ & $6 \%$ \\
\hline No contesta & $\%$ & $\%$ & $0 \%$ & $\%$ & $\%$ & $\%$ & $\%$ & $\%$ & $\%$ & $\%$ & $\%$ & $\%$ \\
\hline No sabe & $\%$ & $\%$ & $\%$ & $\%$ & $\%$ & $4 \%$ & $\%$ & $8 \%$ & $1 \%$ & $\%$ & $\%$ & $0 \%$ \\
\hline (N) & .200 & .200 & .200 & .200 & .200 & .200 & .200 & .200 & .200 & .200 & .200 & .400 \\
\hline
\end{tabular}

Fonte: Elaboración propia con base en el análisis online disponible en Latinobarómetro (2015).

Por ende, no resulta un dato de extrañar, que al momento de observar el nuevo andamiaje constitucional de 1999, en todo el texto - pero especialmente en el artículo 67 -, no se aluda al término partido político, sino que se refiere en términos laxos a organizaciones con fines políticos que se presentan a elecciones.

De forma comparativa, si miramos los tres casos aquí analizados, es posible señalar que sin lugar a dudas, la última década del siglo XX y el inicio del nuevo siglo, fue un rango temporal donde la representación política se puso en revisión, algunas veces bajo el diagnóstico de una crisis, de una metamorfosis, de un desalineamiento o inclusive de un desinterés generalizado en la política.

Ahora bien, si se pone estos casos en comparativa con el resto de la región, el panorama no es homogéneo, porque se identifican dos grandes grupos de países: por un lado, están Uruguay, Chile, Argentina y Perú, donde - a pesar de ciertas oscilaciones en el viraje de siglo - la opinión de los ciudadanos manifiesta que los partidos son centrales y necesarios para la democracia; y por el otro, un grupo donde están los tres casos analizados aquí junto a Colombia, Paraguay y, en menor medida, Brasil, que experimentan, en los primeros años del siglo XXI, una situación diferente: los partidos políticos y la democracia ya no son dos elementos necesariamente vinculados.

Aunque, en general, el final de la primera década del siglo XXI ya muestra nuevamente un momento de "retorno" de la centralidad de los partidos, las explicaciones sobre los momentos de zozobra del vínculo con la democracia no necesariamente se explica en torno a las tradiciones y las trayectorias de estabilidad/inestabilidad que históricamente experimentaron los sistemas de partidos en cada uno de os países, sino más bien en torno a la modalidad en la que los ciudadanos procesaron políticamente las limitaciones de la matriz de sociabilidad de 
tipo neoliberal orientada al mercado y en qué medida, tal y como sucediera a finales de 1980 inicios de los noventa, los partidos políticos de mayor envergadura lograron sobrevivir o dieron paso a nuevas expresiones sociales y políticas. En este marco, una vez más el darwinismo político permitió sobrevivir el viraje al nuevo siglo a los partidos que supieron readaptarse (como es el caso del peronismo en Argentina, del APRA en Perú, de la continuidad en Chile y Uruguay de los mismo partidos, entre otros casos), mientras que, en los casos que los partidos no pudieron hacerlo, este vació fue claramente un espacio de enorme relevancia para la representación a través de los movimientos sociales, nuevos liderazgos y fuerzas partidarias. Por ende, no resulta difícil comprender que, en aquellos espacios donde la tradición status quista de los partidos tradicionales pereció, nuevas elites y sectores de la sociedad civil pusieran en entredicho los partidos políticos y con ello su rol preponderante en el marco de la democracia, y trataran de expresarlo a través de una reforma constitucional.

\section{Nuevas constituciones, disputa por el sentido y democracias inciertas}

Salvo algunas excepciones, la democracia liberal representativa había sido una realidad esquiva en la región y, por lo tanto, en los albores de la "redemocratización" parecía un rompecabezas sin figura de tapa ni instructivo. Asimismo, este retorno a la democracia se da en paralelo al imperio de la desertificación neoliberal, con lo cual todo tipo de revisión en las antípodas de este paradigma no solo ponía en tensión los principios liberales de la representación, como también de los mecanismos de participación ciudadana.

En el viraje del siglo XX, los tres casos estudiados son un claro ejemplo de revisión del sentido liberal de la democracia, y una pretensión política por incorporar un nuevo sentido. $\mathrm{Si}$, en los apartados anteriores, nos abocamos a señalar la revisión de la representación y participación directa de los ciudadanos, para observar los nuevos sentidos de la democracia en Ecuador, Bolivia y Venezuela, la forma en que la democracia aparece nombrada en las nuevas Constituciones puede ser un buen punto de llegada para repensar lo nuevo de los sentidos que se le adjudican a la democracia.

Por ejemplo, la reciente Constitución Boliviana está centrada en el concepto de "pluralismo nacional" (MAYORGA, 2009, p. 251), como rasgo dominante del modelo de Estado Unitario Social de Derecho Plurinacional Comunitario. Tal y como es posible apreciar desde el primer artículo, el concepto de Estado requiere ser fuertemente adjetivado para satisfacer a la voluntad del legislador. 


\begin{abstract}
Art. 1. Bolivia se constituye en un Estado Unitario Social de Derecho Plurinacional Comunitario, libre, independiente, soberano, democrático, intercultural, descentralizado y con autonomías. Bolivia se funda en la pluralidad y el pluralismo político, económico, jurídico, cultural y lingüístico, dentro del proceso integrador del país (BOLIVIA, 2009).
\end{abstract}

Más adelante, se establece una forma de gobierno democrática, participativa, representativa y comunitaria (Art. 11). En este sentido, reconoce que estos tres adjetivos serán canalizados a partir de diversos mecanismos institucionales: el aspecto directo y participativo estará dado por el referendo, la iniciativa legislativa ciudadana y la revocatoria de mandato, entre otras; por su parte, el rasgo representativo se conserva para la elección de representantes por medio del voto universal, directo y secreto; finalmente, el rasgo comunitario del sistema político boliviano, en la nueva constitución, está dado por la elección, designación o nominación de autoridades y representantes establecidos por medio de normas propias de las naciones y pueblos indígenas.

La nueva Constitución ecuatoriana, adjetiva extensamente en el Artículo 1 al Estado, como constitucional de derechos y justicia, social, democrático, soberano, independiente, unitario, intercultural, plurinacional y laico, que se organiza en forma de república y se gobierna descentralizadamente. Cabe llamar la atención aquí, cómo en el esfuerzo por nominar algo nuevo se apela de diversos calificativos. Por su parte, el sustantivo democracia aparece calificado con adjetivos semejantes a los que aparecen en la Constitución boliviana. A la hora de enmarcar la forma de participación de la ciudadanía en todos los asuntos de interés público, establece que se ejercerá, a través de los mecanismos de la democracia representativa, directa y comunitaria (Art. 95).

En la Constitución venezolana, la palabra democracia no se inscribe como sustantivo, sino como uno de los adjetivos que acompaña al sustantivo Estado. Así, se establece que Venezuela se constituye como un Estado democrático y social de Derecho y de Justicia. En el mismo artículo introductorio, incluye a la democracia como uno entre otros valores superiores del ordenamiento jurídico (Art. 2), como la vida, la libertad, la justicia, la igualdad, la solidaridad, la responsabilidad social, la preeminencia de los derechos humanos, la ética y el pluralismo político. Además de aparecer como sustantivo muy escasamente en el texto constitucional, parece ser considerada una palabra estrecha y austera, que no incluye un conjunto de rasgos que, generalmente, son sobreentendidos al aludir a ella. 
De esta manera, al observar estos procesos en perspectiva histórica, resulta sugerente retomar una pregunta que formulan Paz y Cepeda (2007, p. 293) a la hora de analizar el derrotero de las Constituciones ecuatorianas: “ ¿Tantas constituciones y una democracia representativa frágil?”. Para los autores, por lo menos hasta la Constitución ecuatoriana de 1998, se podía observar una contradicción entre los principios e ideales expresados en las Constituciones y la concreción de dichos ideales en la vida política (PAZ y CEPEDA, 2007, p. 297). Es posible replicar la pregunta y la afirmación anterior más allá del caso ecuatoriano, para dar cuenta de múltiples casos regionales.

Tras tres décadas de iniciados los procesos de democratización y habiendo sorteado múltiples crisis políticas en distintos países, sin comprometer el quiebre del orden constitucional, las perspectivas de la democracia en la región son otras. Aún así, es probable que los múltiples sentidos - viejos y nuevos - que las constituciones confieren a la democracia tarden en configurar realidades históricas estables.

- Juan Bautista Lucca é Doutor em Ciências Sociais pela FLACSO. É Professor de Sistemas Políticos Comparados na Faculdade de Ciência Politica e Relações Internacionais da Universidade Nacional de Rosário e Pesquisador do Conselho Nacional de Investigações Científicas e Técnicas (CONICET) sobre Politica Latinoamericana Comparada. E-mail: juanlucca@hotmail.com.

Cintia Pinillos é Doutoranda em Ciência Política pela Universidade Nacional de Rosário. É Professora de Sistemas Políticos Comparados da Faculdade de Trabalho Social da Universidade Nacional de Entre Ríos e Integrante da equipe de Sistemas Politicos Comparados na Faculdade de Ciência Política e Relações Internacionais da Universidade Nacional de Rosário. Email: cintiapinillos@hotmail.com.

\section{Referências}

ALTMAN, David. Democracia directa en el continente americano: ‘autolegitimación gubernamental o censura ciudadana? Política y Gobierno, v. 12, n. 2, p. 203-232, 2005. 
ANSALDI, Waldo. A mucho viento, poca vela. Las condiciones sociohistóricas de la democracia en América Latina. Una introducción. In: ANSALDI, Waldo (Dir.). La democracia en América Latina, un barco a la deriva. Buenos Aires: Fondo de Cultura Económica, 2007.

BASABE-SERRANO, Santiago. Ecuador: reforma constitucional, nuevos actores políticos y viejas prácticas partidistas. Revista de Ciencia Política, Santiago, v. 29, n. 2, p. 381-406, 2009.

BASABE-SERRANO, Santiago; PACHANO, Símon; MEJÍA ACOSTA, Andrés. La democracia inconclusa: derechos fundamentales, instituciones políticas y rendimientos gubernamentales en Ecuador (1979-2008). Revista de Ciencia Politica, Santiago, v. 30, n. 1, p. 65-85, 2010.

BOLIVIA. Constitución (2009). Constitución Politica del Estado Plurinacional de Bolivia. Bolivia: Versión Oficial, 2009.

COLLIER, David; LEVITSKY, Steven. Democracia con adjetivos. Innovación conceptual en la investigación comparativa. Revista Agora, n. 8, p. 99-122, 1998.

DE SOUSA SANTOS, Boaventura; AVRITZER, Leonardo. Introducción: para ampliar el canon democrático. In: DE SOUZA SANTOS, Boaventura (Ed.). Democratizar la democracia: Los caminos de la democracia participativa. México: Fondo de Cultura Económica, 2004. p. 35-74.

HAGOPIAN, Frances. Democracia y representación política en América Latina en los años noventa ¿pausa, reorganización o declinación? In: MAINWARING, Scott; LÓPEZ, Ernesto. Democracias: discusiones y nuevas aproximaciones. Quilmes: Universidad Nacional de Quilmes, 2000. p. 265321.

HOLSTON, James. Citizenship in Disjunctive Democracies. In: TULCHIN, Joseph; RUTHENBURG, Meg (Eds.). Citizenship in Latin America. Londres: Lynne Rienner Publishers, 2007. p. 75-94.

KORNBLITH, Miriam. Democracia directa y revocatoria de mandato en Venezuela. In: LISSIDINI, Alicia et al. (Coord.). Democracia directa en América Latina. Buenos Aires: Prometeo, 2008. p. 117130.

LATINOBARÓMETRO. Análisis online. Madrid, 2015. Disponible en: <http://www.latinobarometro.org/latOnline.jsp>. Acceso en: 20 ago. 2015.

LAZARTE, Jorge. ¿Son necesarios los partidos políticos? La alternativa de las Agrupaciones ciudadanas y de Pueblos indígenas. In: DOMINGO, Pilar (Org.). Bolivia: fin de un siglo y nuevas perspectivas políticas (1993-2003). Barcelona: Bellaterra, 2006. p. 93-117.

LECHNER, Norbert. Los patios interiores de la democracia: subjetividad y política. México: Fondo de Cultura Económica, 1988.

LECHNER, Norbert. El debate sobre Estado y Mercado. Nueva Sociedad, Buenos Aires, n. 121, set.out. 1992.

LISSIDINI, Alicia. Democracia directa Latinoamericana: riesgos y oportunidades. In: LISSIDINI, Alicia et al. (Coord.). Democracia directa en América Latina. Buenos Aires: Prometeo, 2008. p. 1362.

LUCCA, Juan Bautista; HERNÁNDEZ CRUZ, Cecilia. El diverso y difícil tránsito de la justicia en América Latina. Revista Cuarta Época, v. 1, n. 11, p. 207-233, 2013.

MAYORGA, Fernando. Democracia participativa en Bolivia: representación, legitimidad y proceso político decisional. In: LISSIDINI, Alicia et al. (Coord.). Democracia directa en América Latina. Buenos Aires: Prometeo, 2008. p. 221-241. 
MAYORGA, Fernando. Antinomias. El azaroso camino de la reforma política. Cochabamba: CESUUMSS, 2009.

MONEDERO, Juan Carlos. El gobierno de las palabras. Política para tiempos de confusión. España: Fondo de Cultura Económica, 2011.

MONEDERO, Juan Carlos. ¿Posdemocracia? Frente al pesimismo de la nostalgia, el optimismo de la desobediencia. Nueva Sociedad, Buenos Aires, n. 240, p. 68-86, jul.-ago. 2012.

MORLINO, Leonardo. Explicar la calidad democrática: ¿qué tan relevantes son las tradiciones autoritarias? Revista de Ciencia Política, Santiago, v. 27, n. 2, p. 3-22, 2007.

NEGRETTO, Gabriel. Paradojas de la reforma constitucional en América Latina. Journal of Democracy en Español, v. 1, n. 1, p. 38-54, 2009.

PACHANO, Simón. El territorio de los partidos. Ecuador, 1979-2002. In: KORNBLITH, Miriam et al. (Orgs.). Partidos políticos en la región andina: entre la crisis y el cambio. Lima: International Institute for Democracy and Electoral Assistance (IDEA) y Ágora Democrática, 2004. p. 71-91.

PACHANO, Simón. Democracia directa en Ecuador. In: LISSIDINI, Alicia et. al. (Coord.). Democracia directa en América Latina. Buenos Aires: Prometeo, 2008. p. 145-158.

PAZ, Juan; CEPEDA, Miño. Ecuador: democracia con tensiones, sociedad con desatenciones. In: ANSALDI, Waldo (Dir.). La democracia en América Latina, un barco a la deriva. Buenos Aires: Fondo de Cultura Económica, 2007. p. 287-312.

PÉREZ LIÑÁN, Aníbal. Juicio Político al Presidente y nueva inestabilidad política en América Latina. Buenos Aires: Fondo de Cultura Económica, 2009.

RAMOS JIMÉNEZ, Alfredo. Venezuela. El ocaso de una democracia bipartidista. Nueva Sociedad, Buenos Aires, n. 161, p. 35-42, maio-jun. 1999.

RAMOS JIMÉNEZ, Alfredo. Partidos y sistemas de partidos en Venezuela. In: CAVAROZZI, Marcelo; MEDINA, Juan Abal (Comps.). El asedio a la politica. Los partidos latinoamericanos en la era neoliberal. Rosario: Homo Sapiens Ediciones, 2002. p. 381-409.

ROBERTS, Kenneth. El sistema de partidos y la transformación de la representación política en la era neoliberal. In: CAVAROZZI, Marcelo; MEDINA, Juan Abal (Comps.). El Asedio a la política. Los partidos políticos latinoamericanos en la era neoliberal. Rosario: Rosario, 2002. p. 55-76.

ROMERO BALLIVIÁN, Salvador. Democracia directa en Bolivia. In: LISSIDINI, Alicia et al. (Coord.). Democracia directa en América Latina. Buenos Aires: Prometeo, 2008. p. 159-170.

VENEZUELA. Constitución (1961). Constitución de Venezuela. Venezuela: Versión Oficial, 1961.

ZOVATTO, Daniel. Las instituciones de la democracia directa a nivel nacional en América Latina. Un balance comparativo: 1978-2007. Revista de Derecho Electoral, n. 4, p. 1-51, 2007.

Texto recebido em 23 de fevereiro de 2016. Aprovado em 16 de março de 2016. 